OPEN ACCESS

Edited by:

Dean E. Morbeck,

Fertility Associates, New Zealand

Reviewed by:

Panagiotis Drakopoulos,

University Hospital Brussels, Belgium

Wellington P. Martins,

SEMEAR fertilidade, Brazil

*Correspondence:

Qianqian Zhu

Qianqianzhu1988@126.com

Specialty section:

This article was submitted to

Reproduction,

a section of the journal

Frontiers in Physiology

Received: 11 March 2020

Accepted: 10 July 2020

Published: 14 August 2020

Citation:

Zhu Q, Lin J, Gao H, Wang N, Wang $B$ and Wang $Y$ (2020) The Association Between Embryo Quality, Number of Transferred Embryos and Live Birth Rate After Vitrified Cleavage-Stage Embryos and Blastocyst Transfer.

Front. Physiol. 11:930. doi: 10.3389/fphys.2020.00930

\section{The Association Between Embryo Quality, Number of Transferred Embryos and Live Birth Rate After Vitrified Cleavage-Stage Embryos and Blastocyst Transfer}

\author{
Qianqian Zhu*, Jiaying Lin, Haoyuan Gao, Ningling Wang, Bian Wang and Yun Wang \\ Department of Assisted Reproduction, Shanghai Ninth People's Hospital Affiliated to Jiao Tong University School \\ of Medicine, Shanghai, China
}

Objective: The single-embryo transfer (SET) is the recommended approach to improve the live birth rate and reduce the complications related with multiple pregnancies. However, the physicians generally chose to transfer two embryos when the embryo quality decreased. The effect on the in vitro fertilization (IVF) or intracytoplasmic sperm injection (ICSI) outcomes following the transfer of a poor-quality embryo (PQE) along with a good-quality embryo (GQE) has been explored. However, previous studies were limited by the fresh embryo transfer cycles or the small sample size.

Methods: A retrospective cohort study was performed among 26,676 women (the mean age was 31.72 years) undergoing first frozen embryo transfer (FET) from January 2011 to December 2017. Patients were grouped into five subgroups, including SET with one GQE (SET-GQE, 2235 patients for cleavage-stage embryo transfer and 756 patients for blastocyst transfer), SET with one PQE (SET-PQE, 148 patients for cleavage-stage embryo transfer and 362 patients for blastocyst transfer), double-embryo transfer with two GQE (DET-2GQE, 20,461 patients for cleavage-stage embryo transfer and 519 patients for blastocyst transfer), double-embryo transfer (DET) with one GQE plus one PQE (DET-GQE+PQE, 1541 patients for cleavage-stage embryo transfer and 266 patients for blastocyst transfer), and DET with two PQE (DET-2PQE, 228 patients for cleavage-stage embryo transfer and 160 patients for blastocyst transfer). Multivariable logistic regression models were performed after controlling for other potential confounders to estimate the effect of number and quality of transferred embryos on pregnancy outcomes.

Result: Although the live birth rate was significantly higher after DET-GQE+PQE compared with SET-GQE for cleavage-stage embryo transfer [574 of 1541 (37.25\%) vs. 571 of $2235(25.55 \%)]$, no significant difference was found between DET-GQE+PQE and SET-GQE for blastocyst transfer [143 of 266 (53.76\%) vs. 325 of 756 (42.99\%)]. However, DET-GQE+PQE also had the highest multiple live births in both cleavage-stage embryo transfer [134 of $1541(8.70 \%)]$ and blastocyst transfer [46 of 266 (17.29\%)]. 
The live birth rate after SET-PQE significantly decreased in comparison with SET-GQE [cleavage-stage embryo transfer: 18 of 148 (12.16\%) vs. 571 of 2235 (25.55\%); blastocyst transfer: 107 of $362(29.56 \%)$ vs. 325 of 756 (42.99\%)] and significantly increased after DET-2GQE compared with SET-GQE [cleavage-stage embryo transfer: 9357 of $20,461(45.73 \%)$ vs. 571 of 2235 (25.55\%); blastocyst transfer: 313 of 519 (60.31\%) vs. 325 of $756(42.99 \%)]$. The live birth rate was also not different between DET-2PQE and SET-GQE for cleavage-stage embryo transfer and blastocyst transfer [cleavage-stage embryo transfer: 75 of 228 (32.89\%) vs. 571 of 2235 (25.55\%); blastocyst transfer: 74 of 160 (46.25\%) vs. 325 of 756 (42.99)].

Conclusion: In order to minimize the risk of multiple births, the data from this study did not support transferring DET with a GQE plus a PQE compared with SET with a GQE, especially for blastocyst transfer. However, the proportion of patients older than 35 years was small (12.07\% for patients aged 36-39 years and $7.31 \%$ for patients 40 years or older), which limited the generalization of these results to other population.

Keywords: embryo quality, number of embryo transfer, live birth rate, vitrification, frozen embryo transfer

\section{INTRODUCTION}

The development of clinical and laboratory techniques over decades has not only improved the live birth rate after assisted reproduction technology (ART) but also increased the multiple pregnancy rate, which increased the obstetric complication and adverse neonatal outcomes (Neubourg et al., 2002; McLernon et al., 2010). As the ultimate goal of ART is the birth of a healthy child with no maternal complication, the single-embryo transfer (SET) is the recommended approach to achieve this goal and reduce the complications related with multiple pregnancies. However, embryo quality is one important factor to consider when implementing a successful SET. Embryo quality, based on morphological parameters, is a major predictor for the success of implantation and live birth (Della et al., 2007; Ahlstrom et al., 2011). The study conducted by Oron et al. (2014) reported the clinical pregnancy rate and live birth rate after the single good-quality embryo transfer (GQE) were almost twice as high as those after the single poor-quality embryo (PQE) transfer.

Despite the higher multiple pregnancy rate, the physicians generally chose to transfer two embryos when the embryo quality decreased. Two studies have explored the effect on the in vitro fertilization (IVF) or intracytoplasmic sperm injection (ICSI) outcomes following the transfer of a PQE along with a GQE and found that the clinical pregnancy rate and live birth rate were similar when compared with transfer of two GQEs, but the above studies were all conducted in the fresh cycles (Wintner et al., 2017; Li et al., 2018). Furthermore, Dobson et al. (2018) evaluated the influence of double-embryo transfer (DET) with a PQE plus a GQE on the IVF outcome in the fresh and frozen cycles and reported that the live birth rate did not increase but the multiple birth increased compared with SET. However, this study only included blastocyst transfer and the sample size was small in five subgroups divided by the number and quality of embryos available. Therefore, the impact of DET and SET on IVF outcome at different grades of embryo quality in the vitrified cleavage and blastocyst stages needed to be further explored in a large sample study.

We conducted this research aiming at exploring the differences in the association between pregnancy outcomes and number of transferred embryos by grades of embryo quality in both vitrified cleavage-stage embryo transfer and blastocyst transfer.

\section{MATERIALS AND METHODS}

\section{Study Population}

In this retrospective cohort study, we included 26,676 women receiving their first frozen embryo transfer (FET) in the time period from January 2011 to December 2017 at the Department of Assisted Reproduction of the Shanghai Ninth People's Hospital affiliated to Jiao Tong University School of Medicine (a large hospital-based tertiary care reproductive center in Shanghai, China). All included women used autologous oocytes, and each woman was included only once in this study. Women with previous fresh or FET were excluded. This study protocol was approved by the Ethics Committee (Institutional Review Board) of the Shanghai Ninth People's Hospital.

The details about ovulation induction and IVF/ICSI procedure, embryo culture and evaluation, freezing and thawing of embryos, and FET all have been detailed in our previous articles (Kuang et al., 2014a,b; Chen et al., 2015; Du et al., 2017). IVF or ICSI was performed depending on the semen quality. Normal fertilization was assessed 16-18 h after insemination/injection. Then, the embryos were subsequently cultured until day 3 or day $5 / 6$.

Cleavage embryos were classified as GQEs (grade I and II embryos) if they had four cells on day 2 or six to eight cells on day 3, with less than $20 \%$ anucleate fragments according to Cummins criteria (Cummins et al., 1986; Reinblatt et al., 2011). Embryos graded III or IV including those that had only two 
cells on day 2, less than six cells on day 3, and no less than $20 \%$ fragmentation were called poor quality. Blastocyst quality was graded on day 5 or 6 according to the degree of blastocoel expansion, inner cell mass (ICM), and trophectoderm (TE) cells (Gardner et al., 2004). Good-quality embryos were defined as those where at least (3) the blastoceles were filling completely $100 \%$ of the embryo, (B) they are loosely grouped with several cells, and (B) several cells formed in the loose epithelium. Lower-than-3BB-quality embryos on day 5 or 6 were defined as PQEs. The allocation to blastocyst- or cleavage-stage embryo transfer depends on the patient's age and the quality and number of cleavage-stage embryos available. Embryo grading was done by two trained embryologists and was verified by another senior embryologist with years of work experience.

After embryo grading, all cleavage-stage embryos and blastocysts were frozen using the vitrification method. In brief, the cryotop carrier system (Kitazato Biopharma Co., Ltd., Japan) was used for vitrification and 15\% (v/v) ethylene glycol, 15\% (v/v) dimethyl sulfoxide, and $0.5 \mathrm{~mol} / \mathrm{l}$ sucrose were used as the cryoprotectant. For warming, 1.0, 0.5, and $0.0 \mathrm{~mol} / \mathrm{l}$ sucrose solutions were used for stepwise cryoprotectant dilution. All vitrification and warming steps were carried out at room temperature except the first warming step, which was at $37^{\circ} \mathrm{C}$. The same vitrification method was employed throughout the whole study period.

Endometrial preparation was performed as previously described (Du et al., 2017). Natural cycle was used for patients with regular menstrual cycles, and hormone therapy cycle or stimulation cycle was used for patients with irregular menstrual cycles. One or two embryos were transferred, and $\mathrm{P}$ supplementation was provided until 8 weeks of gestation if pregnant. Patients were excluded if they had mixed-cleavage-blastocyst-stage embryo transfer.

A live birth was defined as an infant born alive after 24 weeks of gestation who survived more than 28 days. The patients were grouped into cleavage-stage embryo group and blastocyst group according to the development stage of transferred embryos. Then, for each group, five subgroups were formed according to the number and quality of embryos, SET-GQE included patients with single GQE transfer, SET-PQE consisted of patients with single PQE transfer, DET-2GQE involved patients with double GQE transfer, DET-GQE+PQE were those patients with one GQE and one PQE transfer, and DET-2PQE were patients with two PQEs transfer.

\section{Statistical Analysis}

The baseline characteristics were described with mean (standard deviation, SD) for continuous variables and percentage for categorical variables. Comparisons of baseline characteristics between groups were performed with chi-square test or ANOVA where appropriate. The live birth rates across subgroups stratifying by stage of embryo development were calculated. Multivariable logistic regression was performed to explore the effect of different embryo transfer strategies on the live birth for cleavage-stage embryo transfer and blastocyst transfer, respectively, after controlling for potential confounders including maternal age, maternal BMI, infertility type, parity, duration of infertility, causes of infertility, number of two pronuclear (2PN) embryos, endometrial preparation protocol, and endometrial thickness. Results were reported as adjusted odds ratios (aORs) with 95\% confidence intervals (CIs). All statistical analyses were performed by using the two-sided $5 \%$ level of significance and the statistical package Stata, Version 12 (StataCorp, College Station, TX, United States).

\section{RESULTS}

In total, 26,676 patients underwent the first FET (24,613 patients with cleavage-stage embryo transfer and 2063 with blastocysts transfer) in the study period from 2011 to 2017. Among all patients, 2991 patients underwent transfer of single GQE, 510 patients underwent single PQE, 20,980 patients underwent double GQEs, 1807 patients had DET with one GQE plus one PQE, and 388 patients had two PQEs transfer. The patient and treatment characteristics are presented in Table 1. The proportion of patients less than or equal to 30 years of age (46.68\%) was higher in the group with double GQE transfer, and the proportion of patients 35 years of age or greater $(37.06 \%)$ was higher in the group with single PQE transfer. More than $80 \%$ of patients were nulliparous, and the major infertility cause was female factor.

In general, transfer of blastocyst had a significantly higher rate of live birth than did transfer of cleavage-stage embryo (for SET: blastocyst vs. cleavage-stage embryo $38.64 \%$ vs. $24.72 \%$; for DET: blastocyst vs. cleavage-stage embryo $56.08 \%$ vs. $45.01 \%$; Table 2). The multiple live birth rates were also significantly higher for blastocyst transfer compared with cleavage-stage embryo transfer (for SET: blastocyst vs. cleavage-stage embryo $0.81 \%$ vs. $0.29 \%$; for DET: blastocyst vs. cleavage-stage embryo $21.69 \%$ vs. $13.76 \%$ ).

For patients with cleavage-stage embryo transfer, DET-2GQE had the highest live birth rate $(45.73 \%)$, followed by DET-GQE+PQE (37.25\%), DET-2PQE (32.89\%), SET-GQE $(25.55 \%)$, and SET-PQE which had the lowest live birth rate $(12.16 \%)$. This trend also can be found among patients with blastocyst transfer. DET-2GQE also had the highest multiple live births in both cleavage-stage embryo transfer (14.22\%) and blastocyst transfer (26.20\%). In addition, the live birth rate was significantly higher after blastocyst transfer than after cleavage-stage embryo transfer for each of the five embryo transfer strategies.

Table 3 shows the results from multivariate logistic regression on the live birth rate following SET-GQE against SET-PQE, DET-2GQE, DET-GQE+PQE, and DET-2PQE for cleavage-stage embryo transfer and blastocyst transfer, respectively. For both cleavage-stage embryo transfer and blastocyst transfer, after adjusting for other confounders, the results showed that the live birth rate after SET-PQE significantly decreased in comparison with SET-GQE (cleavage-stage embryo transfer: aOR 0.49, 95\% CI 0.28, 0.84; blastocyst transfer: aOR 0.62, 95\% CI 0.46, 0.83), and live birth rate after DET-2GQE significantly increased compared with SET-GQE (cleavage-stage embryo transfer: aOR 1.62, 95\% CI 1.40, 1.89; blastocyst transfer: aOR 1.76, 95\% CI 1.20, 2.57). Although the live birth rate was significantly higher after 
TABLE 1 | Maternal and treatment characteristics by group of embryo transfer.

\begin{tabular}{|c|c|c|c|c|c|c|}
\hline Demographics & $\begin{array}{l}\text { SET-GQE } \\
(n=2991)\end{array}$ & $\begin{array}{l}\text { SET-PQE } \\
(n=510)\end{array}$ & $\begin{array}{l}\text { DET-2GQE } \\
(n=20,980)\end{array}$ & $\begin{array}{c}\text { DET-GQE+PQE } \\
(n=1807)\end{array}$ & $\begin{array}{c}\text { DET-2PQE } \\
(n=388)\end{array}$ & $P$-value \\
\hline Patients with eSET or eDET & $1266(42.33)$ & $170(33.33)$ & $16,135(76.91)$ & $971(53.74)$ & $195(50.26)$ & \\
\hline Maternal age(y), $n(\%)$ & & & & & & $<0.001$ \\
\hline$\leq 30$ & $1080(36.11)$ & $157(30.78)$ & $9793(46.68)$ & $686(37.96)$ & $144(37.11)$ & \\
\hline $31-34$ & 866 (28.95) & $164(32.16)$ & $6521(31.08)$ & $553(30.60)$ & $117(30.15)$ & \\
\hline$\geq 35$ & $1045(34.94)$ & $189(37.06)$ & $4666(22.24)$ & $568(31.43)$ & $127(32.73)$ & \\
\hline Type of infertility, $n$ (\%) & & & & & & 0.271 \\
\hline Primary infertility & $1599(53.46)$ & $264(51.76)$ & 11, $370(54.19)$ & $958(53.02)$ & $193(49.74)$ & \\
\hline Second infertility & $1392(46.54)$ & $246(48.24)$ & $9610(45.81)$ & $849(46.98)$ & $195(50.26)$ & \\
\hline Parity, $n(\%)$ & & & & & & $<0.001$ \\
\hline Nulliparous & 2577 (86.16) & $421(82.55)$ & 18, 862 (89.90) & $1568(86.77)$ & $330(85.05)$ & \\
\hline Pluriparous & $414(13.84)$ & $89(17.45)$ & $2118(10.10)$ & $239(13.23)$ & $58(14.95)$ & \\
\hline Duration of infertility, mean \pm SD & $3.95 \pm 3.07$ & $4.09 \pm 3.10$ & $3.72 \pm 2.71$ & $4.02 \pm 2.91$ & $4.20 \pm 3.02$ & $<0.001$ \\
\hline Infertility causes, $n(\%)$ & & & & & & 0.008 \\
\hline Female factor & $1816(60.72)$ & $294(57.65)$ & 12,725 (60.65) & $1054(58.33)$ & $241(62.11)$ & \\
\hline Male factor & $282(9.43)$ & $49(9.61)$ & $2281(10.87)$ & $194(10.74)$ & $44(11.34)$ & \\
\hline Combined factor & $631(21.10)$ & $102(20.00)$ & $4219(20.11)$ & 377 (20.86) & $70(18.04)$ & \\
\hline Unexplained & $262(8.76)$ & $65(12.75)$ & 1755 (8.37) & $182(10.07)$ & $33(8.51)$ & \\
\hline Stage of embryo development & & & & & & $<0.001$ \\
\hline Cleavage-stage embryo & $2235(74.72)$ & $148(29.02)$ & $20,461(97.53)$ & $1541(85.28)$ & $228(58.76)$ & \\
\hline Blastocyst & $756(25.28)$ & 362 (70.98) & $519(2.47)$ & $266(14.72)$ & $160(41.24)$ & \\
\hline
\end{tabular}

SET, single embryo transfer; DET, double embryos transfer; GQE, good quality embryo; PQE, poor quality embryo; FET: frozen-thawed embryo transfer; eSET, elective SET; eDET, elective DET.

TABLE 2 | Live birth rate in the first FET cycles, stratified by number of embryo transfer, embryo quality, and stage of embryo development.

\begin{tabular}{|c|c|c|c|c|c|c|}
\hline & \multicolumn{3}{|c|}{ Cleavage-stage embryo transfer } & \multicolumn{3}{|c|}{ Blastocyst transfer } \\
\hline & $\begin{array}{l}\text { Number of } \\
\text { cycles }\end{array}$ & $\begin{array}{c}\text { Live birth } \\
\text { rate (\%) }\end{array}$ & $\begin{array}{c}\text { Multiple live } \\
\text { birth rate (\%) }\end{array}$ & $\begin{array}{l}\text { Number of } \\
\text { cycles }\end{array}$ & $\begin{array}{l}\text { Live birth } \\
\text { rate (\%) }\end{array}$ & $\begin{array}{c}\text { Multiple live } \\
\text { birth rate (\%) }\end{array}$ \\
\hline Total & 24,613 & 43.05 & 12.45 & 2063 & 46.63 & 10.37 \\
\hline SET & 2383 & 24.72 & 0.29 & 1118 & 38.64 & 0.81 \\
\hline SET-GQE & 2235 & 25.55 & 0.31 & 756 & 42.99 & 0.79 \\
\hline DET-2GQE & 20,461 & 45.73 & 14.22 & 519 & 60.31 & 26.20 \\
\hline $\mathrm{DET}-\mathrm{GQE}+\mathrm{PQE}$ & 1541 & 37.25 & 8.70 & 266 & 53.76 & 17.29 \\
\hline DET-2PQE & 228 & 32.89 & 6.14 & 160 & 46.25 & 14.37 \\
\hline
\end{tabular}

SET, single embryo transfer; $D E T$, double embryos transfer; GQE, good quality embryo; $P Q E$, poor quality embryo; FET: frozen-thawed embryo transfer.

DET-GQE+PQE compared with SET-GQE for cleavage-stage embryo transfer (aOR 1.25, 95\% CI: 1.04, 1.51), no significant difference was found between DET-GQE+PQE and SET-GQE for blastocyst transfer ( $\mathrm{aOR} 1.42,95 \% \mathrm{CI}$ : 0.93, 2.15). The live birth rate was also not different between DET-2PQE and SET-GQE for cleavage-stage embryo transfer and blastocyst transfer (cleavage-stage embryo transfer: aOR 1.10, 95\% CI 0.78, 1.55; blastocyst transfer: aOR 1.09, 95\% CI 0.67, 1.76).

\section{DISCUSSION}

This large retrospective cohort study showed that DET with a GQE plus a PQE increased the live birth rate for cleavage-stage embryo transfer but did not increase the live birth rate for blastocyst transfer when compared with SET with a GQE during the first FET treatments. Furthermore, DET with a GQE plus a PQE increased multiple live births for both cleavage-stage embryo transfer and blastocyst transfer. Our study indicated that although DET with a GQE plus a PQE was helpful to improve the live birth rate, it led to the increasing risk of multiple live births for cleavage-stage embryo transfer, whereas DET with a GQE plus a PQE was not found to have any benefit in live birth rate, while increasing multiple births for blastocyst transfer. Therefore, our study advises to weigh up the pros and cons before making the clinical decisions about transferring DET with a GQE plus a PQE, especially for blastocyst transfer. 
TABLE 3 | Logistic regression analysis for live birth rate after FET among patients with different embryo transfer strategies.

\begin{tabular}{|c|c|c|c|c|}
\hline & $\begin{array}{l}\text { Unadjusted odds } \\
\text { ratio }(95 \% \mathrm{Cl})\end{array}$ & $P$-value & $\begin{array}{l}\text { Adjusted odds } \\
\text { ratio }(95 \% \mathrm{Cl})\end{array}$ & $P$-value \\
\hline \multicolumn{5}{|c|}{ Cleavage-stage embryo transfer } \\
\hline SET-GQE & 1.00 & & 1.00 & \\
\hline SET-PQE & $0.40(0.24,0.67)$ & $<0.001$ & $0.49(0.28,0.84)$ & 0.009 \\
\hline DET-2GQE & $2.46(2.22,2.71)$ & $<0.001$ & $1.62(1.40,1.89)$ & $<0.001$ \\
\hline DET-GQE+PQE & $1.73(1.50,1.99)$ & $<0.001$ & $1.25(1.04,1.51)$ & 0.018 \\
\hline DET-2PQE & $1.43(1.07,1.92)$ & 0.017 & $1.10(0.78,1.55)$ & 0.578 \\
\hline \multicolumn{5}{|l|}{ Blastocyst transfer } \\
\hline SET-GQE & 1.00 & & 1.00 & \\
\hline SET-PQE & $0.56(0.43,0.73)$ & $<0.001$ & $0.62(0.46,0.83)$ & 0.001 \\
\hline DET-2GQE & $2.01(1.61,2.53)$ & $<0.001$ & $1.76(1.20,2.57)$ & 0.003 \\
\hline DET-GQE+PQE & $1.54(1.16,2.04)$ & 0.003 & $1.42(0.93,2.15)$ & 0.101 \\
\hline DET-2PQE & $1.14(0.81,1.61)$ & 0.450 & $1.09(0.67,1.76)$ & 0.726 \\
\hline
\end{tabular}

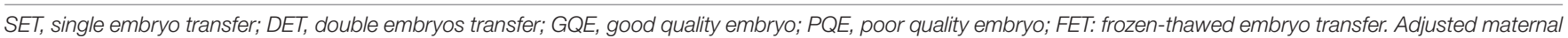

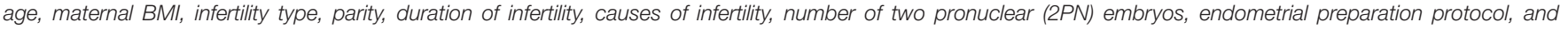
endometrial thickness.

To our knowledge, this was one of the largest retrospective cohort studies exploring the live birth rate after DET with a GQE plus a PQE during the FET treatments. In this study, patients with both the cleavage-stage embryo transfer and blastocyst transfer were enrolled, the results of which could enrich current research in this field. In order to minimize the effect of confounding factors, we only included patients undergoing the first FET and adjusted variables including maternal age, maternal BMI, infertility type, parity, duration of infertility, causes of infertility, number of 2PN embryos, endometrial preparation protocol, and endometrial thickness. However, our study was limited by the retrospective design from the single center; the conclusion from our study needs to be verified in further studies. The proportion of patients older than 35 years was small $(12.07 \%$ for patients aged $36-39$ years and $7.31 \%$ for patients 40 years or older), which also limited the generalization of these results to other population. In addition, the majority of patients in this study underwent cleavage-stage embryo transfer rather than blastocyst transfer. The following reasons can account for the lower proportion of blastocyst transfer in our study. On the one hand, Chinese legislation limited the proportion of blastocyst transfer cycles to control the male birth, which leads to the transfer of cleavage-stage embryos remaining a priority in Chinese IVF centers. On the other hand, extending embryo culture to blastocyst from cleavage stage has been regarded as a tool of embryo selection. Although this procedure improves the live birth rate, it also increases the transfer cancelation rate and diminishes the number of viable embryos for cryopreservation and subsequent frozen-thawed embryo transfer. Previous study has found that the failure to reach the stage of embryo transfer because of poor or arrested embryo development increased in patients seeking blastocyst-stage embryo transfer compared with patients with cleavage-stage embryo transfer (Glujovsky et al., 2016). It has been reported that some cleavage-stage embryos that did not survive extended in vitro culture, however, may continue to develop into viable pregnancies if they were transferred into the uterus on day 3 (Gleicher et al., 2015). So many patients and clinicians choose to cleavage-stage embryo transfer after weighing the pros and cons.

There were different viewpoints on the effect of DET with mixed quality embryos on embryo development and growth. Some authors proposed that the implantation potential of each embryo was independent, and transferring a PQE would not affect the pregnancy and implantation of a GQE. However, the cooperative interaction was proposed to explain that preimplantation embryos could affect the surrounding embryo development by specific released growth factors (Tao et al., 2013). The interaction was related with the embryo quality, and factors from PQE could adversely impact the development of neighboring embryos. In recent years, the decidualized endometrial stromal cells (ESCs) have been a study focus, which could recognize GQE and PQEs and respond selectively to developmentally impaired embryos to prevent its implantation (Weimar et al., 2013). Many previous studies also evaluated the effect of DET with mixed quality embryos on live birth rate by clinical data, and the results were inconsistent. El-Danasouri et al. (2016) reported that transferring a morphologically impaired embryo significantly adversely affected the clinical pregnancy and implantation of the GQE in a retrospective multicenter study. Dobson et al. (2018) finished a prospective study and demonstrated that there exists a non-significant trend of decrease in live birth rate for DET with a GQE plus a PQE compared with SET with a GQE. They attributed the absence of significance to the small sample size and proposed that a PQE may detrimentally impact the implantation of a GQE for blastocyst transfer in both fresh and frozen cycles. Two studies performed in fresh IVF/ICSI cycles found that the live birth rate has no statistically significant differences for DET with mixed quality embryos vs. SET with a GQE or DET with two GQE (Wintner et al., 2017; Li et al., 2018). In contrast to previous studies, our study found the increased live birth rate after DET with a GQE plus a PQE compared with SET with a GQE for vitrified cleavage-stage embryo transfer, but no significant change was found in the live birth rate between DET with a GQE plus a PQE and SET with 
a GQE for blastocyst transfer. Given the conflicted results in the existing research, prospective randomized controlled trials were needed to confirm the relationship.

Consistent with previous studies, our research also showed that the multiple live birth rates per embryo transfer cycle were significantly increased after DET than SET regardless of the transferred embryo quality and the development stage of embryo (Gelbaya et al., 2010; McLernon et al., 2010). Since October 2015, the "universal two-child policy" has been enacted in China (Zeng and Hesketh, 2016; Li et al., 2019). Influenced by this policy, many infertile couples want to have two children with one ART treatment. However, multiple live births were associated with increased obstetrical and neonatal morbidity and mortality. From a healthcare and societal perspective, the cost was also found to be much higher for DET than cumulative SET (Kjellberg et al., 2006). McLernon et al. (2010) also proposed that the difference in live birth rate between SET and DET could be overcome through an additional SET. So we advise these infertile couples anticipating two children to undergo two separate childbirths after SET rather than DET.

In agreement with other studies, the present study found the significantly higher live birth rate after blastocyst transfer than cleavage embryo transfer (Zhu et al., 2013; Glujovsky et al., 2016). The likely explanation for this result was the embryo selection by extending culture to the blastocyst stage, which reduced the number of PQEs, as only those with strong developmental potential survive the challenge of extended culture and reach the blastocyst stage. In our research, the live birth rate for single blastocyst transfer with a GQE was higher than that for double cleavage-stage embryo transfer with mixed quality embryos and double cleavage-stage embryo transfer with two PQE. This result verified that a single blastocyst transfer with a GQE was the preferred recommendation for patients preparing for the first FET.

\section{CONCLUSION}

In conclusion, DET with a GQE plus a PQE increased the live birth rate for cleavage-stage embryo transfer but did not increase the live birth rate for blastocyst transfer when compared with SET with a GQE during the first FET treatments. Furthermore, DET with a GQE plus a PQE increased multiple live births for both

\section{REFERENCES}

Ahlstrom, A., Westin, C., Reismer, E., Wikland, M., and Hardarson, T. (2011). Trophectoderm morphology: an important parameter for predicting live birth after single blastocyst transfer. Hum. Reprod. 26, 3289-3296. doi: 10.1093/ humrep/der325

Chen, H., Wang, Y., Lyu, Q., Ai, A., Fu, Y., Tian, H., et al. (2015). Comparison of live-birth defects after luteal-phase ovarian stimulation vs. conventional ovarian stimulation for in vitro fertilization and vitrified embryo transfer cycles. Fertil. Steril. 103, 1194.e2-1201.e2. doi: 10.1016/j.fertnstert.2015. 02.020

Cummins, J. M., Breen, T. M., Harrison, K. L., Shaw, J. M., Wilson, L. M., and Hennessey, J. F. (1986). A formula for scoring human embryo growth rates in in vitro fertilization: its value in predicting pregnancy and in comparison with cleavage-stage embryo transfer and blastocyst transfer. Therefore, in order to minimize the risk of multiple births, the data from this study did not support transferring DET with a GQE plus a PQE compared with SET with a GQE, especially for blastocyst transfer.

\section{DATA AVAILABILITY STATEMENT}

The datasets generated for this study are available on request to the corresponding author.

\section{ETHICS STATEMENT}

The studies involving human participants were reviewed and approved by the Ethics Committee (Institutional Review Board) of the Shanghai Ninth People's Hospital. Written informed consent for participation was not required for this study in accordance with the national legislation and the institutional requirements.

\section{AUTHOR CONTRIBUTIONS}

QZ contributed to data collection and drafted the manuscript. HG, JL, NW, BW, and YW were responsible for the analysis of data. All authors have read and approved the final manuscript.

\section{FUNDING}

This work was supported by the National Natural Science Foundation of China (grant nos. 81903324, 81771533, 81701523, and 81801527) and the National Key Research and Development Program of China (grant no. SQ2018YFC100163).

\section{ACKNOWLEDGMENTS}

The authors wish to express their thanks to all clinicians and clinical embryologists in the Department of Assisted Reproduction, especially for Dr. Kuang Yanping for his help for the interpretation of the study data.

visual estimates of embryo quality. J. In Vitro Fert. Embryo Transf. 3, 284-295. doi: $10.1007 /$ bf01133388

Della, R. T., Verheyen, G., Papanikolaou, E. G., Van Landuyt, L., Devroey, P., and Van Steirteghem, A. (2007). Developmental stage on day-5 and fragmentation rate on day-3 can influence the implantation potential of top-quality blastocysts in IVF cycles with single embryo transfer. Reprod. Biol. Endocrinol. 5:2. doi: 10.1186/1477-7827-5-2

Dobson, S., Lao, M. T., Michael, E., Varghese, A. C., and Jayaprakasan, K. (2018). Effect of transfer of a poor quality embryo along with a top quality embryo on the outcome during fresh and frozen in vitro fertilization cycles. Fertil. Steril. 110, 655-660. doi: 10.1016/j.fertnstert.2018.05.010

Du, T., Chen, H., Fu, R., Chen, Q., Wang, Y., Mol, B. W., et al. (2017). Comparison of ectopic pregnancy risk among transfers of embryos vitrified on day 3 , day 5 , and day 6. Fertil. Steril. 108, 108-116. 
El-Danasouri, I., Sterzik, K., Rinaldi, L., Pacchiarotti, A., DeSanto, M., and Selman, H. (2016). Effect of transferring a morphologically impaired embryo with a good quality embryo on the pregnancy and implantation rates. Eur. Rev. Med. Pharmacol. Sci. 20, 394-398.

Gardner, D. K., Surrey, E., Minjarez, D., Leitz, A., Stevens, J., and Schoolcraft, W. B. (2004). Single blastocyst transfer: a prospective randomized trial. Fertil. Steril. 81, 551-555. doi: 10.1016/j.fertnstert.2003.07.023

Gelbaya, T. A., Tsoumpou, I., and Nardo, L. G. (2010). The likelihood of live birth and multiple birth after single versus double embryo transfer at the cleavage stage: a systematic review and meta-analysis. Fertil. Steril. 94, 936-945. doi: 10.1016/j.fertnstert.2009.04.003

Gleicher, N., Kushnir, V. A., and Barad, D. H. (2015). Is it time for a paradigm shift in understanding embryo selection? Reprod. Biol. Endocrinol. 13:3. doi: 10.1186/1477-7827-13-3

Glujovsky, D., Farquhar, C., Quinteiro, R. A., Alvarez, S. C., and Blake, D. (2016). Cleavage stage versus blastocyst stage embryo transfer in assisted reproductive technology. Cochrane Database Syst. Rev. 30:CD002118. doi: 10.1002/14651858. CD002118.pub5

Kjellberg, A. T., Carlsson, P., and Bergh, C. (2006). Randomized single versus double embryo transfer: obstetric and paediatric outcome and a costeffectiveness analysis. Hum. Reprod. 21, 210-216. doi: 10.1093/humrep/ dei298

Kuang, Y., Chen, Q., Hong, Q., Lyu, Q., Ai, A., Fu, Y., et al. (2014a). Double stimulations during the follicular and luteal phases of poor responders in IVF/ICSI programmes (Shanghai protocol). Reprod. Biomed. Online 29, 684-691. doi: 10.1016/j.rbmo.2014.08.009

Kuang, Y., Hong, Q., Chen, Q., Lyu, Q., Ai, A., Fu, Y., et al. (2014b). Luteal-phase ovarian stimulation is feasible for producing competent oocytes in women undergoing in vitro fertilization/intracytoplasmic sperm injection treatment, with optimal pregnancy outcomes in frozen-thawed embryo transfer cycles. Fertil. Steril. 101, 105-111. doi: 10.1016/j.fertnstert.2013.09.007

Li, H. T., Xue, M., Hellerstein, S., Cai, Y., Gao, Y., Zhang, Y., et al. (2019). Association of China's universal two child policy with changes in births and birth related health factors: national, descriptive comparative study. $B M J$ 366:14680. doi: 10.1136/bmj.14680

Li, J., Du, M., Zhang, Z., Guan, Y., Wang, X., Zhang, X., et al. (2018). Does a poor-quality embryo have an adverse impact on a good-quality embryo when transferred together? J. Ovarian Res. 11:78. doi: 10.1186/s13048-018-0452-6

McLernon, D. J., Harrild, K., Bergh, C., Davies, M. J., and de Neubourg, D. (2010). Clinical effectiveness of elective single versus double embryo transfer: meta-analysis of individual patient data from randomised trials. $B M J$ 341:c6945. doi: 10.1136/bmj.c6945

Neubourg, D. D., Mangelschots, K., Van Royen, E., Vercruyssen, M., Ryckaert, G., and Valkenburg, M. (2002). Impact of patients' choice for single embryo transfer of a top quality embryo versus double embryo transfer in the first IVF/ICSI cycle. Hum. Reprod. 17, 2621-2625. doi: 10.1093/humrep/17.10.2621

Oron, G., Son, W. Y., Buckett, W., Tulandi, T., and Holzer, H. (2014). The association between embryo quality and perinatal outcome of singletons born after single embryo transfers: a pilot study. Hum. Reprod. 29, 1444-1451. doi: 10.1093/humrep/deu079

Reinblatt, S. L., Ishai, L., Shehata, F., Son, W. Y., Tulandi, T., and Almog, B. (2011). Effects of ovarian endometrioma on embryo quality. Fertil. Steril. 95, 2700-2702. doi: 10.1016/j.fertnstert.2011.03.002

Tao, T., Robichaud, A., Mercier, J., and Ouellette, R. (2013). Influence of group embryo culture strategies on the blastocyst development and pregnancy outcome. J. Assist. Reprod. Genet. 30, 63-68. doi: 10.1007/s10815-012-9892-x

Weimar, C. H., Macklon, N. S., Post, U. E., Brosens, J. J., and Gellersen, B. (2013). The motile and invasive capacity of human endometrial stromal cells: implications for normal and impaired reproductive function. Hum. Reprod. Update 19, 542-557. doi: 10.1093/humupd/dmt025

Wintner, E. M., Hershko-Klement, A., Tzadikevitch, K., Ghetler, Y., Gonen, O., Wintner, O., et al. (2017). Does the transfer of a poor quality embryo together with a good quality embryo affect the In Vitro Fertilization (IVF) outcome? J. Ovarian Res. 10:2. doi: 10.1186/s13048-016-0297-9

Zeng, Y., and Hesketh, T. (2016). The effects of China's universal two-child policy. Lancet 388, 1930-1938. doi: 10.1016/S0140-6736(16)31405-2

Zhu, L., Xi, Q., Zhang, H., Li, Y., Ai, J., and Jin, L. (2013). Blastocyst culture and cryopreservation to optimize clinical outcomes of warming cycles. Reprod. Biomed. Online 27, 154-160. doi: 10.1016/j.rbmo.2013.04.006

Conflict of Interest: The authors declare that the research was conducted in the absence of any commercial or financial relationships that could be construed as a potential conflict of interest.

Copyright (c) 2020 Zhu, Lin, Gao, Wang, Wang and Wang. This is an open-access article distributed under the terms of the Creative Commons Attribution License (CC BY). The use, distribution or reproduction in other forums is permitted, provided the original author(s) and the copyright owner(s) are credited and that the original publication in this journal is cited, in accordance with accepted academic practice. No use, distribution or reproduction is permitted which does not comply with these terms. 